\title{
Single mode injection seeded Nd:YAG oscillator with an adjustable pulse length between 20 and 50 ns
}

\author{
L. Cabaret and C. Drag ${ }^{\mathrm{a}}$ \\ Laboratoire Aimé Cotton, CNRS, bâtiment 505, Campus d'Orsay, 91405 Orsay Cedex, France
}

Received: 11 July 2006 / Accepted: 25 October 2006

Published online: 20 December 2006 - (c) EDP Sciences

\begin{abstract}
Highly stable single axial mode operation is obtained in a pulsed injection seeded oscillator with an adjustable pulse length from $20 \mathrm{~ns}$ to more than $50 \mathrm{~ns}$, at a constant output energy. The adjustment principle is described experimentally and analysed theoretically. The peak to peak time jitter added to a slow time drift is of the order of 1 nanosecond for a 20 nanoseconds pulse length.
\end{abstract}

PACS. 42.55.-f Lasers - 42.60.-v Laser optical systems: design and operation - 42.60.Lh Efficiency, stability, gain, and other operational parameters

\section{Introduction}

Long pulse single mode lasers are interesting for obtaining both high resolution and high peak power. When nonlinear effects have to be used to give access to new laser frequencies, the best pulse length roughly ranges from ten to a hundred nanoseconds $([1,2]$ in the case of optical parametric oscillator); in many cases, such a duration may give rise to enough peak power without needing a high output energy. If the resolution is only limited by the pulse duration (Fourier limited), the fundamental laser source will have - for a Gaussian pulse shape - a linewidth of around 20 to $4 \mathrm{MHz}$. This resolution is useful for various high-resolution spectroscopic applications where continuous-wave (cw) counterparts have insufficient power. Long pulses are easily produced by a high repetition rate, moderate gain, Nd:YAG laser, for instance. A certain pulsewidth tuning is possible by changing the repetition rate of the laser [3]. However, for low repetition rate applications (i.e. when the pulses have to be amplified up to the $100 \mathrm{~mJ}$ level or more), this solution is certainly not optimized. Only a small part of the pulses is useful and the average power is too much high, leading to a quite low efficiency and to possible thermal problems. In this prospect, we have built a single axial mode, TEM $_{00}$ Nd:YAG oscillator, with a pulse width tuning possibility ranging from 20 to $50 \mathrm{~ns}$. This driver is adapted to a low repetition rate $(20 \mathrm{~Hz})$ flashlamp pumped amplifier delivering more than $100 \mathrm{~mJ}$ of output energy. In a multipass scheme (two or four passes), the energy of the driver may be as low as a fraction of millijoule in order to extract most of the energy of a standard Nd:YAG flashlamp pumped laser head (QUANTEL model SF410-07A). The measured depolar-

\footnotetext{
a e-mail: cyril.drag@lac.u-psud.fr
}

ization during the amplification with a two pass scheme is less than $1 \%$. The amplified output beam is planed to be first frequency doubled and subsequently sent to pump either a single mode optical parametric oscillator (OPO) or a titanium sapphire laser. To extend the frequency domain, a part of the fundamental beam can be used for nonlinear mixing with the OPO (or Ti:Sa) beam. In our case, one application of the system will be the "photodetachment microscopy" [4] which requires single longitudinal mode excitation (less than $1 \mathrm{mK}$ ).

\section{Experimental set-up}

The scheme of the oscillator is given Figure 1. The cavity has a stable linear geometry with a plane - concave pair of mirrors. The radius of curvature of the concave mirror ( 1 meter) and the effective cavity length ( 0.17 meter) lead to a stability parameter $g_{1} g_{2}$ of about 0.83 , in a region of the stability diagram which is a little bit sensitive to intracavity thermal lens variations. Compactness reasons do not allow us to operate in the best stability region, around $g_{1} g_{2}=\frac{1}{2}$. However, in our configuration, the misalignment sensitivity is not to high.

The amplifying medium is formed by a half-cylinder of Nd:YAG (4 mm diameter). The cylindrical part is fine grounded to eliminate possible parasitics emission modes and the rear plane face is $R_{\max }$ coated at the pump wavelength $(808 \mathrm{~nm})$ to assure a double pass for the diode pumping beam [5]. The main advantages of this geometry are the simplicity of the diode positioning and the rather good gain distribution. However, it is certainly not the best solution for obtaining an optimized overlapping between the cavity mode and the pumped volume. The 


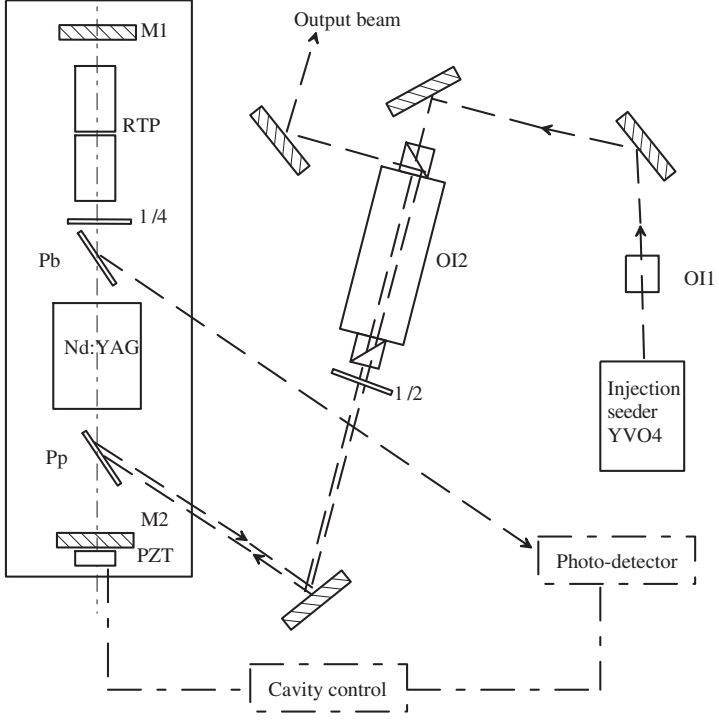

Fig. 1. Experimental Setup. OI1: micro-isolator, OI2: optical isolator, M1 and M2: cavity mirrors, Pb: Brewster plate, Pp: polarizor plate, PZT: piezo actuator.

rod is pumped by a stack of four laser diode bars emitting a total maximum power of 240 watts (SDL 3231-A4). The fundamental $\mathrm{TEM}_{00}$ mode is selected using a $1.1 \mathrm{~mm}$ hole giving a measured radius at the waist: $w_{0}=0.45$ $\pm 0.05 \mathrm{~mm}$. We measure the beam quality factor of the seeded Nd:YAG for two pulse durations (15 ns and $30 \mathrm{~ns}$ ) and find a $M^{2}$ value better than 1.2 . We observe that the beam quality is lightly better for the longer pulse duration. The output coupling is realized through reflection on a dielectric Brewster plate polarizer. The electro-optic Qswitch is composed of a pair of crossed axes RTP crystals $(3 \times 3 \times 10 \mathrm{~mm}$ from the company Cristal Laser $)$, a layout which is quite insensitive to thermal variations. For single mode operation, the cavity is injection seeded by a small home made single axial mode $\mathrm{Nd}: \mathrm{YVO}_{4} \mathrm{cw}$ laser $[6,7]$. The beam is introduced through the coupling polarizer. To completely eliminate the perturbations due to back reflected light from the linear cavity, a $60 \mathrm{~dB}$ isolation is provided by a cascade of two Faraday isolators. One of these isolators acts as a separator between the cw input beam and the pulsed output beam. The other is a micro-isolator with magnetooptical crystal film. A Brewster silica plate is inserted between the Nd:YAG rod and the quater wave plate. In most of the situations, that Brewster plate rejects a high contrast interference cw signal which is useful for locking purposes, as described below. In this arm, the pulsed component is weak enough to avoid the destruction or the complete blinding of the photodiode.

\section{Principle of the pulsewidth adjustment}

In order to adjust the pulse length in a large range without affecting the output energy, three parameters are free: (i) the gain of the amplifying medium, which is managed through the power and the duration of the pump pulse,

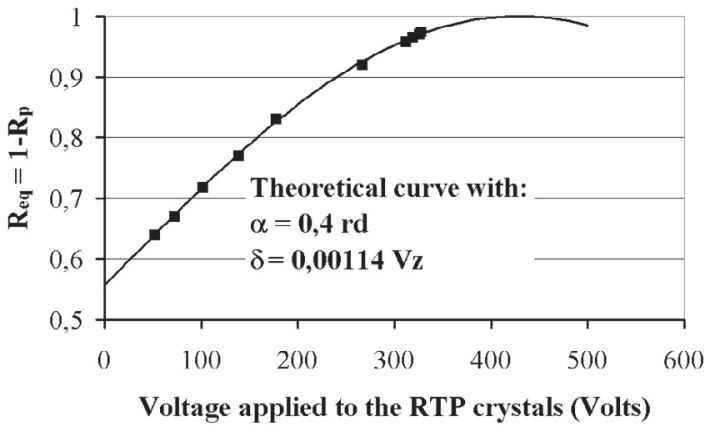

Fig. 2. Equivalent reflectivity of the polarizing output coupler as a function of the voltage applied to the Q-switch crystals. The experimental points (square) correspond to the $R_{p}$ parameters introduced in the simulation to obtain the best fitting curve with $\alpha=0.4$ rd and $\delta=0.00114 \mathrm{~V}$.

(ii) the voltage applied to the Q-switch crystals (RTP), (iii) the orientation of the quater wave plate. These last two parameters control the polarization state inside the cavity and consequently the output coupling reflectivity and the losses on the Brewster silica plate. The main interest of the setup is that it is possible to easily vary the laser pulse width while keeping constant the output energy by a fine adjustment of the three parameters. We can note here that, due to the low average power delivered by the pumping diodes, the thermal effects inside the cavity remain always very weak and do not change the stability parameters of the cavity even under substantial variations of the pumping energy.

Practically, the quater waveplate is not necessary to achieve the coupling adjustment but it is useful to have only to apply a high voltage pulse for $\mathrm{Q}$-switching. In fact, the coupling adjustment could be achieved using a two steps voltage sequence applied to the Q-switch. However, with the use of a waveplate, the voltage remains to zero during the pumping pulse and a simple low voltage edge is needed $(\approx 300 \mathrm{~V})$ to switch on the laser. So, after a first adjustment of the cavity in the gain switched regime at the highest pumping power available, the waveplate is rotated up to just turn off the laser emission. The pulse duration and the energy are then adjusted by varying alternatively the pumping power and the Q-switch voltage. This is described in Figure 2. At the maximum pumping power and without any voltage, we observe the complete turn off for a waveplate angle of $0.4 \mathrm{rad}$ (angle with respect to the incidence plane of the polarizer plate). Starting from this point, by increasing the Q-switch voltage from 0 to $320 \mathrm{~V}$, we are able to vary the equivalent output coupling reflectivity from 0.55 to $1 . R_{e q}$ is a reflectivity equivalent to that of a standard mirror in the transmission mode. The theoretical curve is obtained from Appendix A5 and the experimental points correspond to the parameters introduced in the simulation to obtain the fitting curve of Figure 3 .

A numerical simulation has been done, employing an usual one point set of rate equations for describing the laser dynamics corresponding to our experimental 


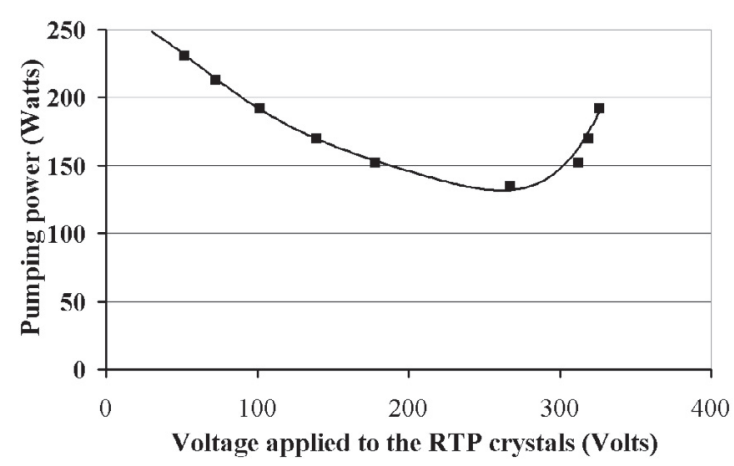

Fig. 3. Pumping power as a function of the voltage applied to the RTP crystals. The output energy is constant at $300 \mu \mathrm{J}$ and the theoretical curves are calculated with a constant loss of $5 \%$ per pass.

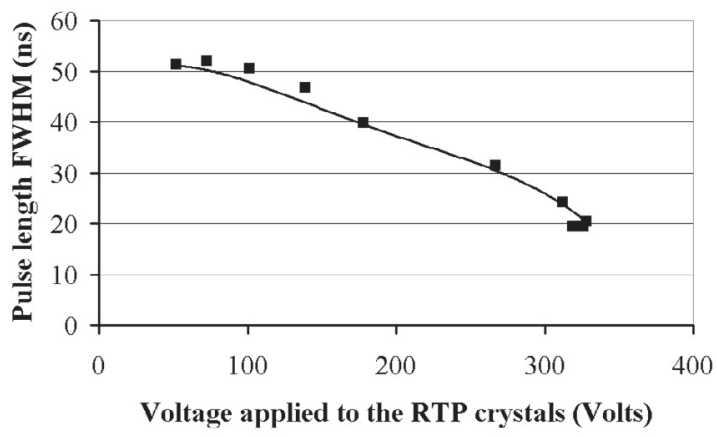

Fig. 4. Output pulse length as a function of the voltage applied to the RTP crystals. The output energy is constant at $300 \mu \mathrm{J}$ and the theoretical curves are calculated with a constant loss of $5 \%$ per pass.

conditions. Considering the output energy and the one pass losses as constants (resp. $300 \mu \mathrm{J}$ and $5 \%$ ), it is clear from Figure 4 that the pulse length $\Delta t$ can be varied between 20 and 50 ns with reasonable values of coupling reflectivity and pumping power (the pumping duration has been fixed at $100 \mu \mathrm{s})$. The pumping power inside the laser mode is calculated by applying a constant coefficient of 0.16 wich represents a rough evaluation of the overlap integral between the pumping beam and the laser mode in the rod. The pumping power in the mode volume has a minimum value of around 20 Watts which corresponds to an optimized output coupling reflectivity $R_{p}$ of $10 \%$ for the chosen energy of $300 \mu \mathrm{J}$. The lowest pulse length is obtained for the highest $\mathrm{Q}$ value, i.e. the lowest output coupling reflectivity in the case of a reflective polarizing plate. Then, on this side, the limitation is due to the intracavity peak power density which may exceed the damage threshold of the components. In the situation described here, the intracavity peak power density at the maximum of a $\mathrm{TEM}_{00}$ Gaussian beam would be about $260 \mathrm{MW} / \mathrm{cm}^{2}$ for a coupling reflectivity $R_{p}$ of $3 \%$. Most of the current optical components can withstand such a power density. On the highest $\Delta t$ side, the limitation is essentially given by the available pumping power. Another interesting fea-

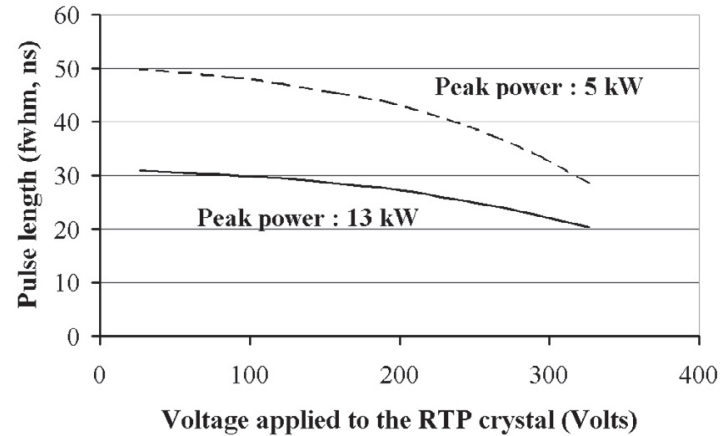

Fig. 5. Calculated pulse length as a function of the voltage applied to the RTP crystals for constant peak power.

ture of the setup is given by the possibility of producing a pulse with a variable length and a constant peak power. However, in a similar domain of output coupling reflectivities, the pulse variation range is weaker as it is shown in Figure 5. For instance, at a constant peak power of $13 \mathrm{~kW}$ (about $300 \mu \mathrm{J}$ at $R_{p}=3 \%$ ), $\Delta t$ varies between 20 and 32 ns and between 29 and 52 ns for a constant peak power of $5 \mathrm{~kW}$ (about $300 \mu \mathrm{J}$ at $R_{p} \cong 35 \%$ ).

In this pulse length range - i.e. 20 to $50 \mathrm{~ns}-$, a higher output energy could be extracted with a larger mode diameter while keeping the same temporal pulse characteristics. The actual mode size is a trade-off between the stability of the cavity, the beam quality and the pumping power available. Considering that we cannot decrease the cavity length, the only way to have access to shorter pulses is to work at a higher gain. Unfortunately, this situation allows us to emit a constant output energy only in a very weak domain of pulse duration. Therefore, to obtain a pulse length in the range from 8 to $20 \mathrm{~ns}$, the duration must be adjusted in a classical manner, for instance by varying the pumping power at the optimized coupling reflectivity.

\section{Single axial mode operation - Injection seeding}

Numerous injection locking techniques can be employed to achieve single axial mode operation. In commercial lasers one often proceeds by minimizing the build up time of the Q-switch pulses [9]. Other methods include the observation of an interference signal through a cavity mirror and by the use of a feedback system to achieve the injection locking [10]. In the first case, the cavity length is adjusted from pulse to pulse and the stability of the locking is sensitive to the perturbations. In the second case, for instance, the cavity length is quickly swept over more than one free spectral range and the laser is fired by a fast electronic system when the resonance is just detected; this method is robust against vibrations but gives a large uncertainty on the Q-switch triggering time. We describe here a rugged and precise technique for injection seeding. In our case, single axial mode operation is realized by injection seeding of a $\mathrm{cw}$ laser beam from a home made low 


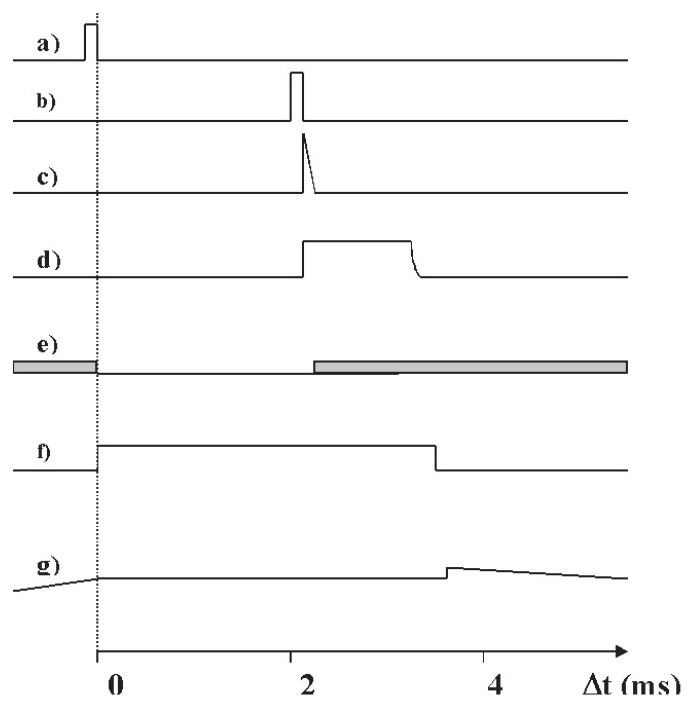

Fig. 6. (a) Trigger pulse. (b) Pumping pulse (100 $\mu$ s duration, not at scale). (c) Q-switch pulse. (d) Saturated photodiode signal of the laser pulse. (e) $1.5 \mathrm{kHz}$ modulation applied to the PZT. (f) Correction applied to the PZT to compensate for $\Delta \varphi$. (g) Error signal maintained to its value at $\Delta t=0$ during about $4 \mathrm{~ms}$.

power $(20 \mathrm{~mW}) \mathrm{Nd}: \mathrm{YVO}_{4}$ oscillator. Its beam is mode matched with the pulsed cavity beam through the polarizing output coupler in order to obtain a perfect locking. When exact alignment and phase locking are achieved, a $60 \mathrm{~dB}$ isolation is necessary to prevent from instabilities. The separation between the input $\mathrm{cw}$ beam and the output pulsed beam is performed inside a first Faraday isolator; a second one, placed just after the seeder, completes the isolation. The plane mirror is mounted on a piezo actuator which undergoes a low amplitude modulation at a frequency of $1.5 \mathrm{kHz}$. An interference signal is conveniently extracted from the intracavity silica Brewster plate and detected by a large area silicon photodiode (UDT 555D). The modulated signal is sent in a phase locking detection system which allows one cavity mode to be locked on the injected frequency. These operations concern the continuous component of the signal; however, since a pulsed component appears at a repetition rate of $20 \mathrm{~Hz}$, three types of perturbations must be overcome: (1) the blindness of the detector by the high peak power optical pulse, (2) a large jitter introduced by the ceramic modulation and (3) a slight optical path drift which detunes the relative phases of the cw and pulsed components.

(1) The peak power of the pulsed part is about $10^{3}$ times higher than the power of the $\mathrm{cw}$ beam but fortunately much lower than the damage threshold of the detector. Therefore, when a correct modulated signal is obtained by placing an attenuator or adjusting the gain, the pulsed signal saturates during almost 2 milliseconds. The large perturbation that results in the gain loop can be suppressed by maintaining the demodulated signal to its value just before the pulse thanks to a sample and hold element.
(2) The weak amplitude modulation applied continuously to the ceramic is sufficient to produce an appreciable shot to shot phase shift. In these conditions, if one tries to lock the cavity mode on to the injected frequency, a large jitter (typically equivalent to the pulse length) is observed that evidently cannot be compensated by the gain loop. This deleterious effect is completely suppressed when the modulation is stopped about 2 microseconds before the Q-switch triggering.

(3) Locking is achieved by using the interference signal produced by the seeder beam between the pulses. When the pumping pulse and, later on, the Q-switch pulse are applied, the rountrip optical path of the cavity is modified due to the combined action of the pumping thermal deposition and of the index variation of the RTP crystals. The phase difference between the two situations - before and during the pulse - can be described in terms of frequency shift of the cavity resonant mode. The frequency shift due to the temperature increase during the pumping pulse has been evaluated in a similar configuration [8] and is less than $2 \mathrm{MHz}$ in our setup. The frequency shift due to the variation of the crystal index, when the Qswitch voltage is applied, is estimated by calculating the optical path variation for the resonant low loss plane of polarisation (incidence plane of the output coupling polarizer). Neglecting the piezoelectric effect which is very low in RTP, the round trip optical path variation $\Delta L$, with the pair of crossed crystals geometry, is simply given by: $\Delta L=\frac{\lambda}{2 \pi} \Delta \varphi$. Where $\Delta \varphi$ is the global phase variation, for the the light component Ex polarized in the reference plane, when the electric field is applied. Since the phase variation is weak $(\Delta \varphi<2 \pi)$, it can be found directly by calculating the evolution of the $a_{11}$ coefficient when the voltage increases from 0 to the working value of $200 \mathrm{~V}$ (see Appendix A4). For the experimental values of $\alpha=0.4 \mathrm{rd}$ and $\delta=0.228 \mathrm{rd}$ (resp. the angle of the quarter waveplate axis with respect to the $x$ axis and the dephasing of the Q-switch due to the electric field) we find $\Delta \varphi=0.662 \mathrm{rd}$ and $\Delta L=0.112 \mu \mathrm{m}$. This corresponds to a frequency shift $\Delta \nu$ of the resonant mode of about $93 \mathrm{MHz}$, much more than the thermal shift. A correction of $\Delta L / 2=0.056 \mu \mathrm{m}$ has then to be applied to the position of a cavity mirror in order to keep the resonant mode in phase with the injected mode. Knowing the properties of the PZT ceramic, the voltage needed to obtain this correction is only $1.4 \mathrm{~V}$, not far from the measured value of $1.5 \mathrm{~V}$. Figure 6 is a summary of the time delays employed to overcome these three perturbations.

The long interruption time of 4 milliseconds around each pulse does not allow to work at a high repetition rate which is limited, up to now, to $25 \mathrm{~Hz}$. Before the laser pulse, the perturbation time of 2 milliseconds is mainly due to the response time of the piezoceramic to the square pulse that is applied for the frequency shift correction. One way to decrease this response time would be to employ a lighter mirror assembly. At a repetition rate of $20 \mathrm{~Hz}$, a very stable behaviour is observed. The long term stability has been simply characterized by observing the oscilloscope trace with an infinite persistence 
(Tektronix TDS 784D). When the cavity is perfectly seeded, the build up time of the pulse is minimum; any locking default is seen as a thickening or a jump of the trace. The peak to peak temporal jitter is given by the thickness of the trace, with the oscilloscope triggered by the HV Q-switch pulse. For a pulse length of $20 \mathrm{~ns}$, we measured a total peak to peak jitter added to a residual slow time drift of $1 \mathrm{~ns}$ during a one hour run. It is a rather good jitter for an injection seeded oscillator. The value of the bandwidth is roughly $30 \mathrm{MHz}$ and the long term stability is $+/-100 \mathrm{MHz}$ during a few hours because of a weak oscillation of the temperature of the $\mathrm{Nd}: \mathrm{YVO}_{4} \mathrm{cw}$ laser. This two values were obtained with a commercial Angstrom WS-U lambda-meter. In the future, we want to more precisely characterize the bandwidth of the pulsed laser by the use of an optical heterodyne measurement [11, $12]$.

\section{Conclusion}

We have analysed the possibility of obtaining an adjustable pulse length emission from a low repetition rate Nd:YAG oscillator. Without modifying the beam properties, we are able to adjust the pulse length between $20 \mathrm{~ns}$ and more than 50 ns while keeping constant the output energy at $300 \mu \mathrm{J}$ in our scheme. In a more reduced range, a pulse length adjustment is also possible while keeping the peak power constant. We have demonstrated a rugged method to stabilize the injection seeding of a low repetition rate oscillator: hours of operation without any mode hop are obtained at a repetition rate of $20 \mathrm{~Hz}$.

Future developments with this oscillator concern: (1) the multipass amplification using a solid state phase conjugation (i.e. Brillouin effect in D-LAP), (2) the use of an electro-optic crystal to modulate the interference signal in order to increase the repetition rate to at least $100 \mathrm{~Hz}$, (3) the use of other laser materials such $\mathrm{Nd}: \mathrm{GdVO}_{4}$ at $1.083 \mathrm{~nm}$.

The authors would like to acknowledge Professor Pierre Camus, who is now retired, for his assistance in performing the early setup.

\section{Appendix A: Jones matrix formalism (JM)}

\section{A1 Reference coordinate system}

As describe in Figure 7, we choose a direct coordinate system linked to the propagation vector $\mathbf{k}$ of the beam inside the cavity, so that $\mathbf{k}$ remains parallel to $z$ and positive along a roundtrip. The polarization state is observed through the $x y$ plane looking towards the light source. Following this conventional description, the end of the electric vector rotates clockwise for a right-handed wave $\left(\sigma^{+}\right)$and counterclockwise for a left-handed wave $\left(\sigma^{-}\right)$.

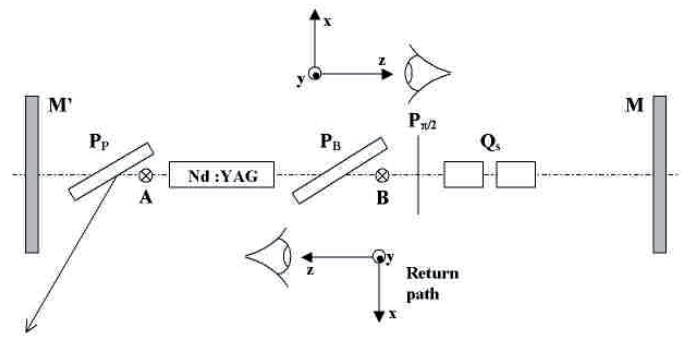

Fig. 7. Experimental setup and coordinate system to do calculations with Jones matrix formalism.

This convention implies, for the return path:

1- to apply a rotation operator (a $\pi$ rotation around the $y$ axis) to the Jones vector incident on the mirror M:

$$
M_{\pi}=\left[\begin{array}{cc}
-1 & 0 \\
0 & 1
\end{array}\right]
$$

2- to invert the sign of the rotation angles, marked out in the $x y$ plane, for the JM of the components.

In addition, the sense of light propagation inside a component is indicated by an oriented arrow on the top of its JM.

\section{A2 Equivalent JM for the two crystals RTP Q-switch}

RTP is an electrooptic biaxial crystal with crystal symmetry $\left(\mathrm{mm}^{2}\right)$. We consider a RTP crystal with a reference crystalline set of main axes $(X, Y, Z)$. The propagation vector of the laser beam inside the cavity, $\mathbf{k}$, is collinear to the crystalline $Y$ axis.

The JM for a single birefringent crystal, of length $l$, which axes $X$ and $Z$ are parallel to the reference coordinate axes - resp. $x$ and $y-$ is written as:

$$
B_{C}=\left[\begin{array}{cc}
\exp -i \Phi_{X} & 0 \\
0 & \exp -i \Phi_{Z}
\end{array}\right]
$$

where $\Phi_{X}$ is the phase retardation for the wave component polarized along the $X$ axis and $\Phi_{Z}$ is the phase retardation for the wave component polarized along the $Z$ axes. If the corresponding refractive indices are respectively $n_{X}$ and $n_{Z}$, we have:

$$
\Phi_{X}=\frac{2 \pi n_{X} l}{\lambda} \text { and } \Phi_{Z}=\frac{2 \pi n_{Z} l}{\lambda} .
$$

In the chosen orientation, when an electric field $E_{Z}=V / d$ is applied along the $Z$ axis of the crystal, the indices are modified as following:

$$
\begin{aligned}
& n_{X V}=n_{X} \mp \frac{n_{X}^{3}}{2} r_{13} \frac{V}{d} \\
& n_{Z V}=n_{Z} \mp \frac{n_{Z}^{3}}{2} r_{33} \frac{V}{d} .
\end{aligned}
$$

The minus sign applies for an electric field oriented in the sense of the polar $Z$ axis, the plus sign applies in the 
opposite case. No mixed terms appear, and the orientation of the principal axes is unchanged. This gives the modified phases:

$$
\begin{aligned}
& \Phi_{X V}=\frac{2 \pi l}{\lambda}\left(n_{X} \mp \frac{n_{X}^{3}}{2} r_{13} \frac{V}{d}\right)=\Phi_{X} \mp \Delta \Phi_{X} \text { and } \\
& \Phi_{Z V}=\frac{2 \pi l}{\lambda}\left(n_{Z} \mp \frac{n_{Z}^{3}}{2} r_{33} \frac{V}{d}\right)=\Phi_{Z} \mp \Delta \Phi_{Z} .
\end{aligned}
$$

So that the new JM of the crystal, in the presence of an electric field $E_{Z}$, can be written as:

$$
\vec{B}_{C}^{ \pm}=\left[\begin{array}{cc}
\exp -i\left(\Phi_{X} \mp \Delta \Phi_{X}\right) & 0 \\
0 & \exp -i\left(\Phi_{Z} \mp \Delta \Phi_{Z}\right)
\end{array}\right] .
$$

To express the Jones vectors in the crystal system of coordinates, in which the JM is diagonal, the reference coordinate system may need to be rotated. A clockwise rotation of angle $\alpha$ about the $z$ axis, is described using the standard rotation matrix $R(\alpha)$ :

$$
R(\alpha)=\left[\begin{array}{cc}
\cos \alpha & \sin \alpha \\
-\sin \alpha & \cos \alpha
\end{array}\right]
$$

Provided that the $y$ and $Z$ axes are always kept of the same sense and direction by employing the adequate rotations, we have the following relations:

$$
\vec{B}_{C}^{+}=\overleftarrow{B}_{C}^{+} \text {and } \vec{B}_{C}^{-}=\overleftarrow{B}_{C}^{-}
$$

In the birefringence compensated scheme, the two RTP crystals have exactly the same length and their $Z$ axes make a relative angle of $90^{\circ}$. In order to have additive electro-optical contributions, the electric field $E_{Z}$ must be applied in the opposite sense on each crystal. Consequently, the JM for the Q-switch component is calculated as following:

$$
\vec{Q}_{S}=R\left(-\frac{\pi}{2}\right) \times \vec{B}_{C}^{+} \times R\left(\frac{\pi}{2}\right) \times \vec{B}_{C}^{-} .
$$

Which gives the simple form:

$$
\begin{aligned}
\vec{Q}_{S}= & \exp \left[-i\left(\Phi_{X}+\Phi_{Z}\right)\right] \\
& \times\left[\begin{array}{cc}
\exp \left[-i\left(\Delta \Phi_{Z}-\Delta \Phi_{X}\right)\right] & 0 \\
0 & \exp \left[-i\left(\Delta \Phi_{X}-\Delta \Phi_{Z}\right)\right]
\end{array}\right]
\end{aligned}
$$

As expected, if no electric field is applied to the crystal, $\vec{Q}_{S}$ is the JM of an isotropic phase retarder of optical thickness $\left(n_{X}+n_{Z}\right) l$. In the presence of the electric field $E_{Z}$, the induced phase retardation is:

$$
2\left(\Delta \Phi_{Z}-\Delta \Phi_{X}\right)=\frac{2 \pi l}{\lambda} \times \frac{V_{Z}}{d} \times n_{Z}^{3} \times\left(\frac{n_{X}^{3}}{n_{Z}^{3}} r_{13}-r_{33}\right) .
$$

A quater wave retardation is thus obtained for a voltage amplitude:

$$
V_{Z \frac{\pi}{2}}=\frac{\lambda d}{4 n_{Z}^{3} l\left(\frac{n_{X}^{3}}{n_{Z}^{3}} r_{13}-r_{33}\right)}
$$

giving the following JM:

$$
\vec{Q}_{S \frac{\pi}{2}}=\exp \left[-i\left(\Phi_{X}+\Phi_{Z}\right)\right] \times \exp \left[i \frac{\pi}{4}\right] \times\left[\begin{array}{cc}
1 & 0 \\
0 & -i
\end{array}\right] .
$$

In our cavity scheme, complete Q-switching requires the use of a quater wave phase retardation of the electrooptical component. For a beam propagation along the $Y$ crystal axis, the beam polarization direction at the entrance of the crystals must be $45^{\circ}$ with respect to the $X$ and $Z$ axes. So, with such an orientation of the crystal pair, the JM of the Q-switch becomes, in the cavity reference frame:

$$
\vec{Q}_{S}=R\left(\frac{\pi}{4}\right) \times \vec{B}_{C}^{+} \times R\left(-\frac{\pi}{2}\right) \times \vec{B}_{C}^{-} \times R\left(\frac{\pi}{4}\right) .
$$

Which gives explicitly:

$$
\begin{aligned}
\vec{Q}_{S}= & \exp \left[-i\left(\Phi_{X}+\Phi_{Z}\right)\right] \\
& \times\left[\begin{array}{cc}
\cos \left(\Delta \Phi_{Z}-\Delta \Phi_{X}\right) & -i \sin \left(\Delta \Phi_{Z}-\Delta \Phi_{X}\right) \\
-i \sin \left(\Delta \Phi_{Z}-\Delta \Phi_{X}\right) & \cos \left(\Delta \Phi_{Z}-\Delta \Phi_{X}\right)
\end{array}\right]
\end{aligned}
$$

and for the return path:

$$
\overleftarrow{Q}_{S}=R\left(\frac{\pi}{4}\right) \times \vec{B}_{C}^{-} \times R\left(-\frac{\pi}{2}\right) \times \vec{B}_{C}^{+} \times R\left(\frac{\pi}{4}\right)
$$

giving:

$$
\begin{aligned}
\overleftarrow{Q}_{S}= & \exp \left[-i\left(\Phi_{X}+\Phi_{Z}\right)\right] \\
& \times\left[\begin{array}{cc}
\cos \left(\Delta \Phi_{Z}-\Delta \Phi_{X}\right) & i \sin \left(\Delta \Phi_{Z}-\Delta \Phi_{X}\right) \\
i \sin \left(\Delta \Phi_{Z}-\Delta \Phi_{X}\right) & \cos \left(\Delta \Phi_{Z}-\Delta \Phi_{X}\right)
\end{array}\right]
\end{aligned}
$$

\section{A3 Jones matrices of the other intracavity components}

\section{A3.1 Jones matrix of the quater wave plate}

The axes of the quater wave plate may have an arbitrary orientation with respect to the reference axes of the cavity. If $\alpha$ is the angle between the slow axis of the plate and the $x$ axis of the cavity, the JM of the plate are expressed by:

$$
\vec{P}_{\frac{\pi}{2}}=\left[\begin{array}{ll}
A & C \\
C & B
\end{array}\right]
$$

and:

$$
\overleftarrow{P}_{\frac{\pi}{2}}=\left[\begin{array}{cc}
A & -C \\
-C & B
\end{array}\right]
$$

with

$A=\exp \left[i \frac{\pi}{4}\right] \times\left(\cos ^{2} \alpha-i \sin ^{2} \alpha\right)$

$B=\exp \left[i \frac{\pi}{4}\right] \times\left(\sin ^{2} \alpha-i \cos ^{2} \alpha\right)$

$C=i \frac{\sqrt{2}}{2} \sin 2 \alpha$. 


\section{A3.2 Jones matrix of the Brewster $\mathrm{SiO}_{2}$ plate}

In our case, the Brewster $\mathrm{SiO}_{2}$ plate is oriented such that its incidence plane is parallel to the $x z$ plane of the cavity. In this condition, the JM of the plate is:

$$
P_{B}=\left[\begin{array}{cc}
\tan \theta_{B} & 0 \\
0 & 2 \sin ^{2} \theta_{B}
\end{array}\right] \times\left[\begin{array}{cc}
\cot \theta_{B} & 0 \\
0 & 2 \cos ^{2} \theta_{B}
\end{array}\right]
$$

The matrix on the right represents the input interface [air $\left.-\mathrm{SiO}_{2}\right]$ at the Brewster's angle $\theta_{B}$, and the matrix on the left the output interface $\left[\mathrm{SiO}_{2}-\right.$ air $]$. This reduces to:

$$
P_{B}=\left[\begin{array}{cc}
1 & 0 \\
0 & \sin ^{2} 2 \theta_{B}
\end{array}\right]=\left[\begin{array}{cc}
1 & 0 \\
0 & \frac{4 n^{2}}{\left(1+n^{2}\right)^{2}}
\end{array}\right] .
$$

And considering the orientation of the plate, the relation: $\vec{P}_{B}=\overleftarrow{P}_{B}$ is always valid

\section{A3.3 Jones matrix of the Brewster dielectric polarizing plate}

As in the preceding case, the plate is oriented such that its incidence plane is parallel to the $x z$ plane of the cavity.

One face of this plate has a polarizing dielectric coating, which can be considered as a partial polarizer. The second face is a non coated Brewster face. The overall JM for the component is then:

$$
\begin{aligned}
\vec{P}_{P} & =\overleftarrow{P}_{P}=\left[\begin{array}{cc}
\tan \theta_{B} & 0 \\
0 & 2 \sin ^{2} \theta_{B}
\end{array}\right] \times\left[\begin{array}{cc}
p_{1} \cot \theta_{B} & 0 \\
0 & 2 p_{2} \cos ^{2} \theta_{B}
\end{array}\right] \\
& =\left[\begin{array}{cc}
p_{1} & 0 \\
0 & p_{2} \sin ^{2} 2 \theta_{B}
\end{array}\right] .
\end{aligned}
$$

\section{A4 Matrix of the system at point A}

For a complete analysis of the polarization behaviour of the light beam inside the cavity, we look for polarization states that remain unchanged after a round trip through the cavity. The Jones matrix method allows us to find polarization eigenstates for the resonator by multiplying together the matrices of the elements with respect to their orientation in the cavity reference frame. However, in our case, the problem can be simplified considering that the Brewster polarizing plate is a perfect polarizer (i.e. $p_{1}=1$ and $\left.p_{2}=0\right)$. Starting from point $A$ to the right with a beam polarized along the $x$ axis, the polarization state at $A$, after the return, is described by the relation:

$$
\left[\begin{array}{l}
\overleftarrow{E} x \\
\overleftarrow{E} y
\end{array}\right]_{A}=C_{A}\left[\begin{array}{l}
\overrightarrow{1} \\
\overrightarrow{0}
\end{array}\right]
$$

The global JM $C_{A}$ that describes a travel of the light beam through the cavity, starting at point $A$ in the right direction, is then the product:

$$
C_{A}=\overleftarrow{P}_{B} \overleftarrow{P}_{\frac{\pi}{2}} \overleftarrow{Q}_{S} M_{\pi} \vec{Q}_{S} \vec{P}_{\frac{\pi}{2}} \vec{P}_{B}=\left[\begin{array}{ll}
a_{11} & a_{12} \\
a_{21} & a_{22}
\end{array}\right]
$$

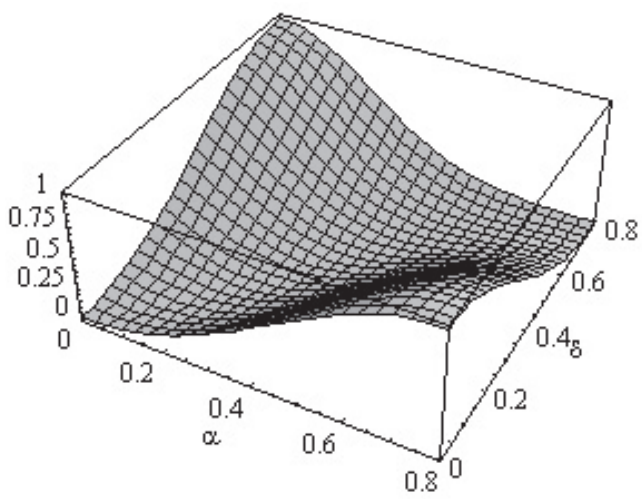

Fig. 8. Reflectivity of the coupling polarizer $R_{p}$ in function of $\alpha$ and $\delta$.

The four elements of $C_{A}$ are calculated using the individual matrices of the optical components; we obtain:

$$
\begin{aligned}
& a_{11}=-\sin 2 \alpha \sin 2 \delta-i \cos 2 \alpha(\cos 2 \delta+\sin 2 \alpha \sin 2 \delta) \\
& a_{12}=-a_{21} \\
& a_{21}=i N\left(\sin 2 \alpha \cos 2 \delta-\cos ^{2} 2 \alpha \sin 2 \delta\right) \\
& a_{22}=N^{2}[\sin 2 \alpha \sin 2 \delta-i \cos 2 \alpha(\cos 2 \delta+\sin 2 \alpha \sin 2 \delta)] \\
& \text { with } \delta=\Delta \Phi_{X}-\Delta \Phi_{Z} \text { and } N=\frac{4 n^{2}}{\left(1+n^{2}\right)^{2}} .
\end{aligned}
$$

\section{A5 Reflectivity of the output coupling polarizer}

The knowledge of the state of polarization at point $A$ permits to calculate the outputcoupling reflectivity through the polarizing Brewster plate. This perfect polarizer reflects the $y$ component of the wave and transmits completely the $x$ component. So, if $I_{x}$ and $I_{y}$ are the intensities of respectively the $x$ and $y$ components in $A$, just before the polarizer, its reflectivity is given by:

$R_{p}=\frac{I_{y A}}{I_{x A}+I_{y A}}=\frac{E_{y A} E_{y A}^{*}}{E_{x A} E_{x A}^{*}+E_{y A} E_{y A}^{*}}=\frac{a_{21} a_{21}^{*}}{a_{11} a_{11}^{*}+a_{21} a_{21}^{*}}$.

After introducing the values for $a_{i j}$, we obtain:

$$
R_{p}=\frac{I}{I+J+L}
$$

with

$$
\begin{aligned}
& I=N^{2}\left(\cos 2 \delta \sin 2 \alpha-\cos ^{2} 2 \alpha \sin 2 \delta\right)^{2} \\
& J=\sin ^{2} 2 \alpha \sin ^{2} 2 \delta \\
& K=\left(\cos 2 \delta \sin 2 \alpha-\cos ^{2} 2 \alpha \sin 2 \delta\right)^{2} \\
& L=\cos ^{2} 2 \alpha(\cos 2 \delta+\sin 2 \alpha \sin 2 \delta)^{2} .
\end{aligned}
$$

We plot $R_{p}$ in Figure 8 .

\section{A6 Losses due to the Brewster plate}

Starting from a completely $x$ polarized beam in $A$, there is no loss for the first path in the Brewster plate. For the return path, the knowledge of the polarization state just before the Brewster plate in $B$, allows us to calculate the reflection losses per path. 


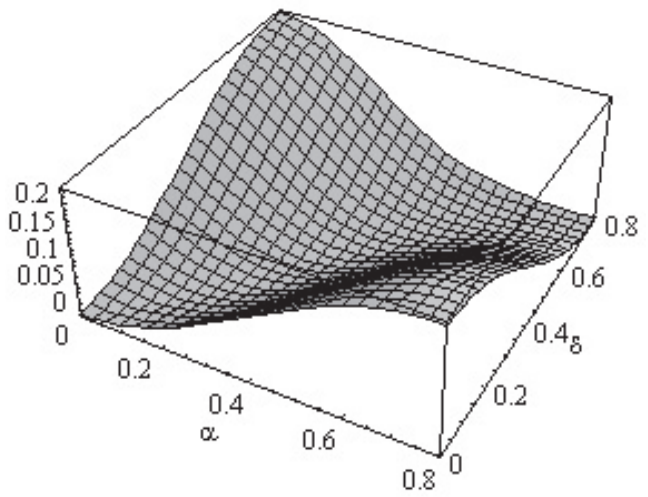

Fig. 9. Losses per roundtrip due to the Brewster plate $L_{b}$ in function of $\alpha$ and $\delta$.

The polarization state in $B$ is obtained by the relation:

$$
\left[\begin{array}{l}
\overleftarrow{E} x \\
\overleftarrow{E} y
\end{array}\right]_{B}=C_{B}\left[\begin{array}{l}
\overrightarrow{1} \\
\overrightarrow{0}
\end{array}\right]
$$

with:

$$
C_{B}=\overleftarrow{P}_{\frac{\pi}{2}} \overleftarrow{Q}_{S} M_{\pi} \vec{Q}_{S} \vec{P}_{\frac{\pi}{2}} \vec{P}_{B}=\left[\begin{array}{ll}
b_{11} & b_{12} \\
b_{21} & b_{22}
\end{array}\right]
$$

The four elements of $C_{B}$ are then:

$$
\begin{aligned}
& b_{11}=a_{11} \\
& b_{12}=a_{12} \\
& b_{21}=a_{21} / N \\
& b_{22}=a_{22} / N .
\end{aligned}
$$

The reflected intensity which corresponds to the losses per roundtrip due to the Brewster plate, is:

$$
L_{b}=1-\frac{I_{x A}+I_{y A}}{I_{x B}+I_{y B}}=1-\frac{a_{11} a_{11}^{*}+a_{21} a_{21}^{*}}{b_{11} b_{11}^{*}+b_{21} b_{21}^{*}} .
$$

After introducing the values for $a_{i j}$ and $b_{i j}$, we obtain:

$$
L_{b}=1-\frac{I+J+L}{J+K+L}
$$

where $I, K$ and $L$ are given above. We plot $L_{b}$ in Figure 9 .

\section{References}

1. S.J. Bronan, R.L. Byer, IEEE J. Quantum Elect. 15, 415 (1979)

2. C. Drag, I. Ribet, M. Jeandron, M. Lefebvre, E. Rosencher, Appl. Phys. B 73, 195 (2001)

3. A.E. Siegman, LASERS (University Science Books, Mill Valley, California, 1986), Chap. 26

4. C. Blondel, C. Delsart, F. Dulieu, Phys. Rev. Lett. 77, 3755 (1996)

5. D. Welford, D.M. Rines, B.J. Dinerman, R. Martinsen, IEEE J. Quantum Elect. 28, 1075 (1992)

6. D.C. Brown, R. Nelson, L. Billings, Appl. Opt. 36, 8611 (1997)

7. G.J. Kintz, T. Baer, IEEE J. Quantum Elect. 26, 1457 (1990)

8. L. Cabaret, J. Philip, P. Camus, IEEE J. Quantum Elect. 36, 1323 (2000)

9. L.A. Rahn, Appl. Opt. 24, 940 (1985)

10. E.S. Fry, Q. Hu, X. Li, Appl. Opt. 30, 1015 (1991)

11. M.S. Fee, K. Danzmann, S. Chu, Phys. Rev. A 45, 4911 (1992)

12. R.T. White, Y. He, B.J. Orr, M. Kono, K.G.H. Baldwin, J. Opt. Soc. Am. B 21, 1577 (2004) 